\title{
Mott transition in one dimension
}

\author{
T. Giamarchi ${ }^{a} *$ \\ ${ }^{a}$ Laboratoire de Physique Des Solides, U.P.S. Bât 510, 91405 Orsay, France
}

I review some of the results on the Mott transition in one dimensional systems obtained in 11 , 3 . I discuss the phase diagram and critical properties of both Mott transitions at fixed filling and upon doping, as well as the dc and ac conductivity. Application of these results to organic conductors is discussed.

\section{Introduction}

The Mott transition is one of the most fascinating phenomenons arising from electron-electron interactions, and occurs in a wide range of materials [4]. In fact two different Mott transitions exist: one can either stay at a given (commensurate) filling and vary the strength of the interactions (I call this transition Mott- $\mathrm{U}$ and it occurs in e.g. vanadium oxides or in organic (quasi-)one dimensional systems), one can also keep the strength of the interaction constant and dope the system to move away from the commensurate density (a Mott- $\delta$ transition, a situation realized in High Tc superconductors or in quantum wires). Although the basic underlying physics behind these transitions is by now well understood it has proved incredibly difficult to tackle it in either $d=2$ or $d=3$ due to our lack of tools to treat strongly interacting systems [5]. In fact nearly all the fine points of the transition, such as the critical properties or the transport properties remain unknown.

One dimension constitute a special case where a rather complete study of the Mott transition can be done. This offers special interest both for theoretical and experimental reasons. From a theoretical point of view, the effect of e-e interactions is particularly strong and leads to a nonfermi liquid state (the so called Luttinger liquid (LL)). One can therefore expect drastic effects on the transport properties of the system. From the experimental point of view, both transitions at constant doping and by varying the doping can be

\footnotetext{
*Laboratoire associé au CNRS. Email: giam@lps.upsud.fr
}

realized, e.g. in organic conductors 6] and quantum wires [7] or Josephson junction networks [B].

Although the thermodynamic properties of the Mott transition were understood a long time ago for the Hubbard model, which was shown to be a Mott insulator at half filling [9 11], very little was known of the Mott- $\delta$ transition and of the transport properties: a parquet treatment gave the effective scattering 12 but was limited to half filling and small (perturbative) interactions, and only the zero frequency conductivity (i.e. the Drude weight) could be computed by Bethe-Ansatz for the particular case of the Hubbard model 13,14]). Recently a complete picture of both Mott transitions as well as a full description of the transport properties $\sigma(\omega, T, \delta)$ for any commensurate filling, both for bosons or fermions, was obtained [1 13$]$. In these proceedings I will review some of these results. No derivation will be given and the reader is referred to [1] 3] for derivation as well as the complete analytical expressions, for the figures presented here. Such a presentation is done in section 2 where umklapp effects are presented and in section 3 where both the critical properties of the Mott transition(s) and the transport properties are examined. In section 1 application of these results to the physics of the organic materials is done.

\section{Lattice effects and umklapp terms}

In the continuum e-e interactions conserve momentum, and thus current, and cannot lead to any finite conductivity as a consequence of Galilean invariance. In the presence of a lattice however the momentum need only to be con- 
served modulo one vector of the reciprocal lattice, and such interaction process (named umklapp process) can lead to finite resistivity. In a fermi liquid, umklapps are responsible for the intrinsic resistivity $\rho(T) \sim T^{2}$.

In one dimension it was rapidly realized 10,11] that umklapps are also responsible for the Mott$\mathrm{U}$ transition at half filling. Away from half filling they are "frozen" due to the mismatch in momentum, and are usually discarded as irrelevant: the system becomes then a perfect metal. However both for the transport properties and to study the Mott- $\delta$ transition it is necessary to have a description of the umklapp processes even for finite doping.

This can be achieved using the so called bosonization representation, that uses that all excitations of a one dimensional system can be described in term of density oscillations [10, 11, 15. The charge properties of a full interacting one dimensional system (excluding umklapp terms) is therefore described by

$H_{0}=\frac{1}{2 \pi} \int d x u_{\rho} K_{\rho}\left(\pi \Pi_{\rho}\right)^{2}+\frac{u_{\rho}}{K_{\rho}}(\nabla \phi)^{2}$

where $\nabla \phi=\rho(x), \rho(x)$ is the charge density and $\Pi$ is the conjugate momentum to $\phi$. All the interaction effects are hidden in the parameters $u_{\rho}$ (the velocity of charge excitations) and $K_{\rho}$ (the Luttinger liquid exponent controlling the decay of all correlation functions). This description (11) is valid for an arbitrary one-dimensional interacting system, provided one uses the proper $u$ and $K$ (in the following I will drop the $\rho$ index). In a general way $K=1$ is the noninteracting point, $K>1$ means attraction whereas $K<1$ means repulsion.

The umklapp process can also be given in terms of boson operators 10,11. In fact umklapps exist not only at half filling but for higher commensurabilities as well by transferring more particles across the fermi surface (such processes are generated in higher order in perturbation theory) [3,16]. For even commensurabilities the Hamiltonian corresponding to the umklapp process is

$H_{\frac{1}{2 n}}=g_{\frac{1}{2 n}} \int d x \cos \left(n \sqrt{8} \phi_{\rho}(x)+\delta x\right)$ where $n$ is the order of the commensurability ( $n=1$ for half filling - one particle per site; $n=2$ for quarter filling - one particle every two sites and so on). The coupling constant $g_{1 / 2 n}$ is the umklapp process corresponding to the commensurability $n$ and $\delta$ the deviation (doping) from the commensurate filling. $g_{\frac{1}{2}}$ corresponds to one particle per site (half filling) For simple models such as the Hubbard model $g_{\frac{1}{2}}=U$, but this does not need to be the case for more general models

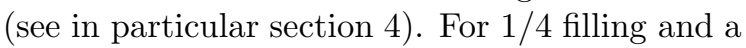
typical interaction $U$ one has $g_{1 / 4} \sim U\left(U / E_{F}\right)^{3}$. Odd commensurability involves spin [16] but can be treated similarly. Similar expressions can be derived for the case of bosons [2]. It is therefore remarkable that in one dimension $H_{0}+H_{\frac{1}{2 n}}$ provides the solution to all Mott transitions, for all systems and all (for particles with spin: even) commensurabilities.

\section{Mott transition(s)}

Let us now examine the physical properties of the Mott transitions close to a commensurability of order $n$ described by $H_{0}+H_{\frac{1}{2 n}}$.

\subsection{Phase diagram}

The Mott- $U$ and Mott- $\delta$ transitions are radically different and lead to the phase diagram shown in Figure 1 .

The Mott-U is of the Kosterlitz Thouless type 10,11 and occurs for a critical value of $K, K_{c}=$ $1 / n^{2}$ [3,16]. For half filling the transition point is the noninteracting one $\left(K_{c}=1\right)$ but for higher commensurability one reaches the Mott insulator only for very repulsive interactions (for example for quarter filling $K_{c}^{U}=1 / 4$ ). In the metallic phase the system is a LL, with finite compressibility and Drude weight. The Mott insulator has a gap in the charge excitations (thus zero compressibility). At the transition there is a finite jump both in the compressibility and Drude weight. The dynamical exponent is $z=1$.

To study the Mott- $\delta$ transition it is useful [1] to map the sine-gordon Hamiltonian $H_{0}+H_{\frac{1}{2 n}}$ to a spinless fermion model (known as massive Thiring model [10,11]), describing the charge excitations (solitons) of the sine-gordon model. The remark- 


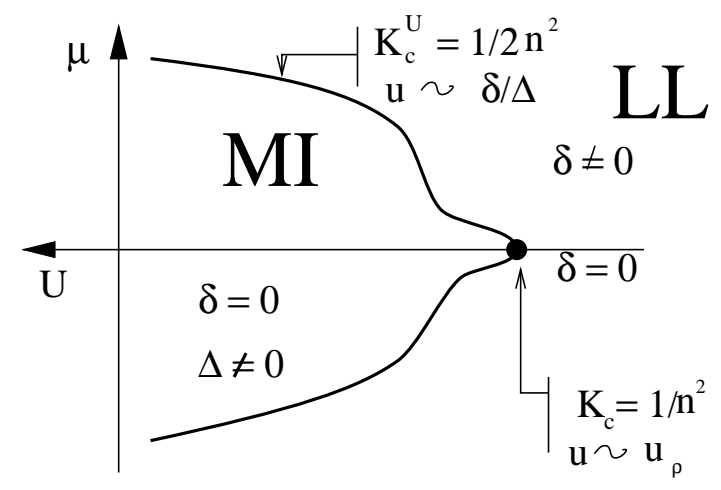

Figure 1. Phase diagram close to a commensurability of order $n(n=1$ for half filling and $n=2$ for quarter filling). $U$ denotes a general (i.e. not necessarily local) repulsion. $\mu$ is the chemical potential and $\delta$ the doping. MI means Mott insulator and LL Luttinger liquid (metallic) phase. The critical exponent $K_{c}$ and velocity $u$ depends on whether it is a Mott- $\mathrm{U}$ or Mott- $\delta$ transition.

able fact is that close to the Mott- $\delta$ transition the solitons become non-interacting, and one is simply led to a simple semi-conductor picture of two bands separated by a gap (see figure 2). This image has to be used with caution since the solitons are only non-interacting for infinitesimal doping (or for a very special value of the initial interaction) and has to be supplemented by other techniques [1. Nevertheless it provides a very appealing description of the LHB and UHB and a good guide to understand the phase diagram and transport properties. The Mott- $\delta$ transition is of the commensurate-incommensurate type. The universal (independent of the interactions) value of the exponents $K_{c}^{\delta}=1 /\left(2 n^{2}\right)$ is half of the one of Mott-U transition. Since at the Mott- $\delta$ transition the chemical potential is at the bottom of a band the velocity goes to zero with doping. This leads to a continuous vanishing of the Drude weight and compressibility. The dynamical exponent is now $z=2$. For more details see [1, 3, 16, 17]. For bosons, the phase diagram of figure 1 is well compatible with numerical results 18] and higher dimensional proposals [19].

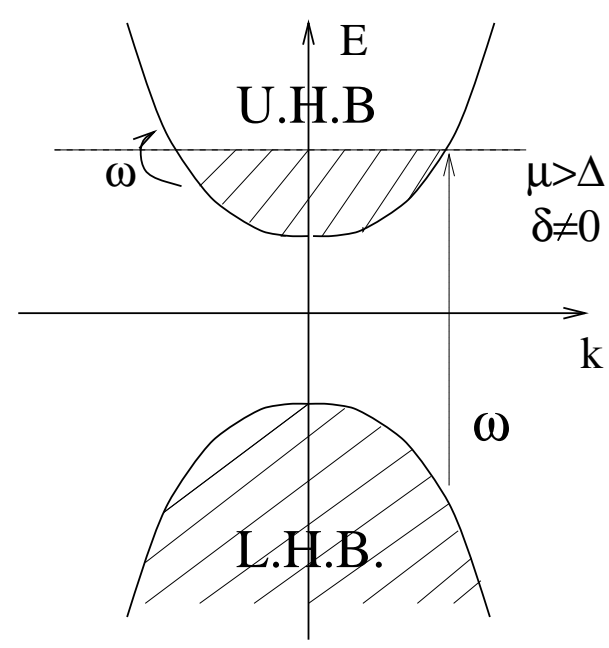

Figure 2. Lower Hubbard band and Upper Hubbard band. This concept can be made rigorous in one dimension by mapping the full interacting system to a massive Thiring model. Optical transitions can be made either within or between the two "bands".

\subsection{Transport properties}

Let us now look at the transport properties. The full conductivity (real and imaginary part) $\sigma(\omega, T, \delta)$ can be found in [1- 3 and we just examine here simple limits.

The ac conductivity (at $T=0$ ) for $\delta=0$ is shown in figure 3. In the Mott insulator $\sigma$ is zero until $\omega$ can make transitions between the LHB and UHB. At the threshold one has the standard square root singularity coming from the density of states (see figure 2). For higher frequencies interactions dress the umklapps and give a nonuniversal (i.e. interaction-dependent) power law-like decay. Such a power law is beyond the reach of the simple noninteracting description of Figure 2.

Away from commensurate filling $(\delta \neq 0)$ the conductivity is shown in Figure 1 (only the case where the half filled system is a MI is shown. For the other case see [2]). Features above the Mott gap are unchanged (the system has no way to know it is or not at half filling at high frequen- 


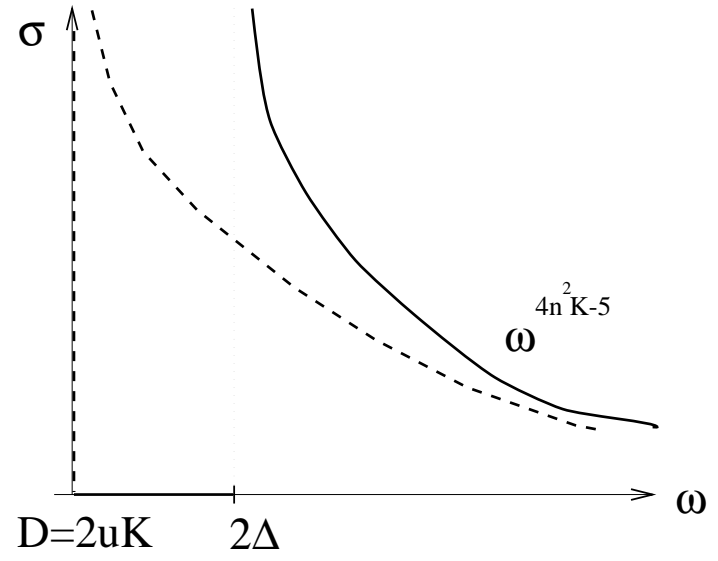

Figure 3. ac conductivity for $\delta=0$ for a commensurability of order $n . \Delta$ is the Mott gap. The full line is the conductivity in the Mott insulator. The dashed one is $\sigma$ in the metallic regime. It contains both a Drude peak of weight $D$ and a regular part.

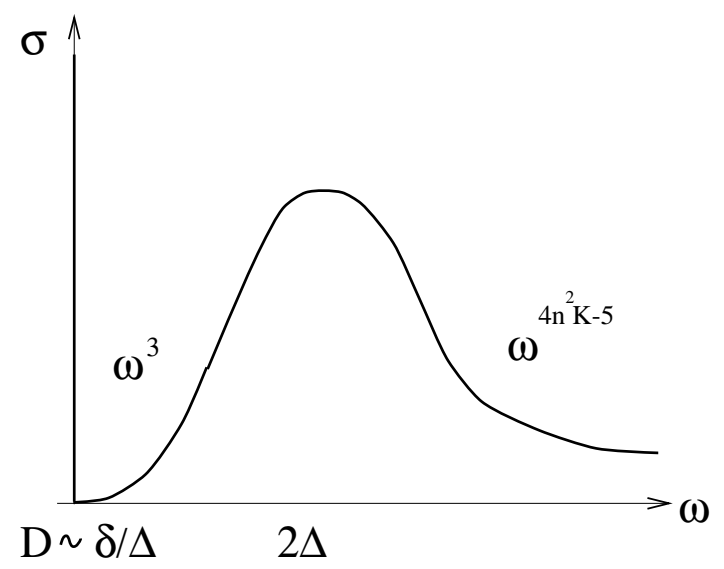

Figure 4. ac conductivity for $\delta \neq 0$ for a commensurability of order $n . \Delta$ is the Mott gap. In addition to the Drude peak of weight $D$ the regular part has two distinct regimes.

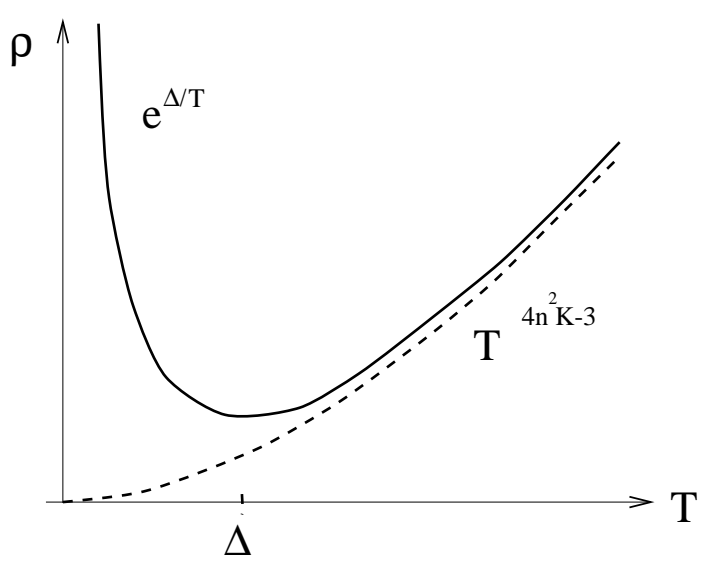

Figure 5. dc conductivity as a function of $T$. Full line is for the Mott insulator, dashed line is in the metallic regime. $\Delta$ is the Mott gap.

cies). The two new features are a Drude peak with a weight proportional to $\delta / \Delta$, and an $\omega^{3}$ absorption [3] at small frequency. Features above the Mott gap come from inter (hubbard)-band transitions whereas they come from intra-band processes below the Mott gap (see figure 2).

The dc conductivity can be computed by the same methods and is shown in figure 5. Here again the dressing of umklapps by the other interactions results in a nonuniversal power law dependence. If the interactions are repulsive enough the resistivity can even increase as a function of temperature well above the Mott gap.

Two universal behavior are expected: at the Mott-U transition one has $\rho(T) \sim T$ and $\sigma(\omega) \sim$ $1 /\left(\omega \ln (\omega)^{2}\right)$, whereas at the Mott- $\delta$ transition due to the different $K_{c}$ one expects $\rho(T) \sim 1 / T$.

All this results are completely general and apply to any one-dimensional systems for which $\Delta$ is smaller than the scale above which all interactions can be treated perturbatively (typically $U)$, a situation that covers most of the experimentally relevant cases (see section 4 ). It is noteworthy that the above results are also valid in the presence not of umklapp processes but of a simple periodic potential (the lattice corresponds itself to a $4 k_{F}$ periodic potential). For a $2 k_{F}$ pe- 


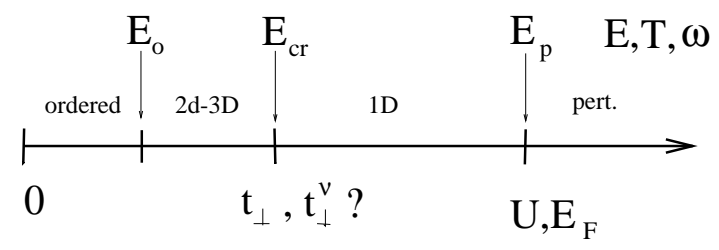

Figure 6. Four important energy regimes for quasi-one dimensional systems. In "pert." everything can be treated perturbatively. In " $1 \mathrm{D}$ " the interactions lead to the one dimensional physics, and hopping from chain to chain is incoherent. In "2D-3D" the hoping between chains is coherent. The system orders in "Ordered".

riodic potential transport properties are similar to the one above with the replacement of $4 n^{2} K_{\rho}$ by $1+K_{\rho}$.

\section{Organic compounds}

The above results have a direct application to organic conductors. These compounds are $1 / 4$ filled by chemistry but due to a slight dimerization of the chain an half filled umklapp $g_{1 / 2} \sim$ $U\left(D / E_{F}\right)$ also exists where $D$ is the dimerization gap, and $U$ a typical strength of the interactions 6]. Since $D / E_{F}$ is quite small the umklapp term is much smaller than the other interactions leading to a quite small Mott gap (see e.g. 20 for a numerical estimation of the parameters). There is also a $1 / 4$ filled umklapp $g_{1 / 4} \sim U\left(U / E_{F}\right)^{3}$, which is as we saw less relevant but can be depending on the typical interaction $U$ much larger in magnitude than $g_{1 / 2}$.

Since the organic conductors are only quasi-one dimensional systems with a perpendicular hopping integral $t_{\perp}$ between the chains one can distinguish various domains in energy scale (temperature or frequency) as shown in figure 6 The most relevant questions being of course: what is the strength of the interactions in these systems, what is the scale for $T_{\text {cr }}$ (the bare $t_{\perp}$ or lower 21 ), and what is the physics below $T_{\mathrm{cr}}$.

In the absence of $t_{\perp}$ one expects therefore these compounds to be Mott insulators. This is the case for the TMTTF family that has indeed a conductivity 22] similar to the one of figure 5 (full line). Indeed if $\Delta>T_{\mathrm{cr}}$, one expects the Mott gap to render the single particle hopping $t_{\perp}$ irrelevant ( $E_{\text {cr }}$ would thus not exist). This family should be described by one-dimensional physics. Further check of this can be provided by examination of the optical (ac) conductivity, and comparing it to figure 3. Measurements of the transverse conductivity would also give information on the relevance of the transverse hopping. Note that the temperature dependence of the dc resistivity and the frequency dependence of the optical conductivity provide a direct measure of the $K_{\rho}$ exponent of the Luttinger Liquid and give therefore crucial information on the importance of interactions in such systems (the optical conductivity has the advantage to be free from thermal expansion problems). A naive fit in TMTTF would give a value of $K_{\rho} \sim 0.8$, widely different from the one of $K_{\rho}=0.3$ extracted from the NMR 23]. A way to get out of this predicament could be that the conductivity is in fact dominated by $1 / 4$ filling umklapp processes till very close to the Mott gap giving

$\rho(T) \sim g_{1 / 2}^{2} T^{4 K-3}+g_{1 / 4}^{2} T^{16 K-3} \sim g_{1 / 4}^{2} T^{16 K-3}(3)$

but this point clearly deserves further investigation.

On the other hand, the TMTSF family shows a rather good metallic behavior with a $T^{2}$ resistivity, indicating the importance of transverse hopping. This is to be expected if $\Delta>E_{\mathrm{cr}}$. There is important controversy on the value of $E_{\mathrm{cr}}$ [24, 25. Regardless of the value of $E_{\mathrm{cr}}$ the physics for $(\omega, T)>E_{\text {cr }}$ will still be controlled by one dimensional effects. Indeed For the TMTSF family the optical conductivity [26] is very well compatible with the figure 4. In particular the optical peak can easily be interpreted in term of the Mott insulator described here. Of course more detailed comparison of the structure above the gap an in particular a check for the power law decay of figure 4 would be worthy to do. The low energy features and in particular the metallic behavior are closer to the doped system rather than the commensurate one. "doping" is not too surprising since if one particle hopping between chains is 
relevant, one expect small deviations to the commensurate filling due to the warping of the Fermi surface. One therefore expect a very small spectral weight in the $\delta(\omega)$ part. Since one has a clear idea of the (purely) one-dimensional conductivity

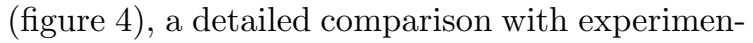
tal data should provide an indication on the value of $E_{\mathrm{cr}}$. The question on whether the physics below $E_{\text {cr }}$ is simply "fermi liquid" like [25] or still retains some features of one-dimensionality and interactions is still open. One way to settle this issue is a detailed examination of the low frequency part of the optical conductivity and measurements of the transverse dc conductivity in this regime. Another way would be to examine the effects of impurities on the dc conductivity. Indeed one expect drastic localization in a one dimensional regime and very weak effects for a FL 27].

\section{Acknowledgments:}

It is a pleasure to thank L. Degiorgi, L.P. Gor'kov, G. Grüner, D. Jérome, A.J. Millis, H.J. Schulz and B.S. Shastry for many interesting discussions.

\section{REFERENCES}

1. T. Giamarchi, Phys. Rev. B 44, 2905 (1991).

2. T. Giamarchi, Phys. Rev. B 46, 342 (1992).

3. T. Giamarchi and A. J. Millis, Phys. Rev. B 46, 9325 (1992).

4. N. F. Mott, Metal-Insulator Transitions (Taylor and Francis, London, 1990).

5. Various mean field theories like slave bosons or $d=\infty$ limit can be applied to study the Mott transition in more than one dimension. See e.g. A. Georges, G. Kotliar, W. Krauth and M. J. Rozenberg Rev. Mod. Phys. 6813 (1996) and references therein.

6. D. Jérome and H. J. Schulz, Adv. Phys. 31, 299 (1982).

7. S. Tarucha, T. Honda, and T. Saku, Sol. State Comm. 94, 413 (1995). S. Tarucha, T. Saku, Y. Tokura, and Y. Hirayama, Phys. Rev. B 47, 4064 (1993).

8. A. van Oudenaarden and J. E. Mooij, Phys. Rev. Lett. 76, 4947 (1996).

9. E. H. Lieb and F. Y. Wu, Phys. Rev. Lett.
20, 1445 (1968).

10. V. J. Emery, in Highly Conducting OneDimensional Solids, edited by J. T. D. et al. (Plenum, New York, 1979), p. 327.

11. J. Sólyom, Adv. Phys. 28, 209 (1979).

12. L. P. Gorkov and I. E. Dzyaloshinski, JETP Lett. 18, 401 (1973).

13. B. S. Shastry and B. Sutherland, Phys. Rev. Lett. 65, 243 (1990).

14. H. J. Schulz, Phys. Rev. Lett. 64, 2831 (1990).

15. F. D. M. Haldane, J. Phys. C 14, 2585 (1981).

16. H. J. Schulz, in "Strongly correlated electronic materials", edited by K. S. Bedell and al. (Addison-Westley, Reading, Massachusetts, 1994).

17. M. Mori, H. Fukuyama, and M. Imada, J. Phys. Soc. Jpn. 63, 1639 (1994).

18. G. G. Batrouni, R. T. Scalettar, and G. T. Zimanyi, Phys. Rev. Lett. 65, 1765 (1990).

19. M. P. A. Fisher, P. B. Weichman, G. Grinstein, and D. S. Fisher, Phys. Rev. B 40, 546 (1989).

20. K. Penc and F. Mila, Phys. Rev. B 50, 11429 (1996).

21. See e.g. D. Boies, C. Bourbonnais and A.-M. S. Tremblay Phys. Rev. Lett 74968 (1995) and references therein.

22. F. Creuzet et al., J. Phys. (Paris) C3, 1099 (1983).

23. P. Wzietek et al., J. Phys. (Paris) 3, 171 (1993).

24. K. Behnia et al., Phys. Rev. Lett. 74, 5272 (1995).

25. L. P. Gor'kov "Non fermi liquid features in Q1D organic conductors due to their proximity to SDW state." this volume.

26. M. Dressel et al., Phys. Rev. Lett. 77, 398 (1996).

27. T. Giamarchi and H. J. Schulz, Phys. Rev. B 37, 325 (1988) and references therein. 